Original Paper http://ajol.info/index.php/ijbcs http://indexmedicus.afro.who.int

\title{
Etude phytochimique et activité antioxydante comparatives des trois variétés de Catharanthus roseus (L.) G. Don
}

\author{
Hèzouwè KAGNOU ${ }^{1}$, Oudjaniyobi SIMALOU ${ }^{1 *}$, Gneiny Whad TCHANI ${ }^{1}$, \\ Sabrina SANVEE ${ }^{1}$, Kokou Agbékonyi AGBODAN ${ }^{2}$, Outéndé TOUNDOU ${ }^{3}$ et \\ Kafui KPEGBA ${ }^{1}$ \\ ${ }^{1}$ Laboratoire de Chimie Organique et des Substances Naturelles (Lab COSNat), \\ Faculté des Sciences, Université de Lomé, 01 BP 1515 Lomé 01, Lomé, Togo. \\ ${ }^{2}$ Laboratoire de Génie des Procédés et Ressources Naturelles (LAGEPREN), Département de \\ Chimie, Université de Lomé, 01 BP 1515 Lomé 01, Lomé, Togo. \\ ${ }^{3}$ Laboratoire de physiologie et de biotechnologie végétale (LPBV), Faculté des Sciences, Université de Lomé, \\ 01 BP 1515 Lomé 01, Lomé, Togo. \\ *Auteur correspondant ; E-mail: jacobsimalou@yahoo.fr
}

\section{RESUME}

Catharanthus roseus (L.) G. Don, est une plante médicinale largement utilisée en tradithérapie contre le diabète, l'hypertension, le cancer et les effets du stress oxydatif. Elle existe en trois variétés ("rosea", "alba" et "hybride") selon la couleur des fleurs. L'étude actuelle porte sur le criblage phytochimique et l'activité antioxydante in vitro (DPPH et FRAP) de l'extrait hydro-éthanolique des trois variétés. Les tests ont été effectués sur les extraits des feuilles, fleurs et racines. On a noté la présence des alcaloïdes, phénols, flavonoïdes, tanins, saponines, stérols, terpènes et quinines. Cependant, l'absence des sucres réducteurs, des flavonoïdes et des saponines dans les racines ainsi que les quinines dans les feuilles a été relevée. L'analyse comparative par piégeage du radical DPPH a montré que les extraits des fleurs et racines de l'hybride étaient plus actifs, respectivement de $130,023 \pm 3,2$ et $131,189 \pm 1,8 \mathrm{mg} \mathrm{EqQ} / \mathrm{g}$. Une tendance différente a été observée dans les extraits des feuilles où la meilleure valeur DPPH de 65,746 $\pm 2,5 \mathrm{mg} \mathrm{EqQ} / \mathrm{g}$ est attribuée à "rosea" alors que celle

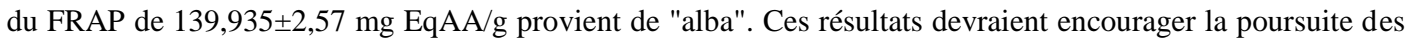
travaux sur l'identification et la caractérisation de nouveaux composés bioactifs sélectivement abondant pour une meilleure valorisation de Catharanthus roseus sur le plan de phytomédicaments.

(C) 2020 International Formulae Group. All rights reserved.

Mots clés : Catharanthus roseus, variétés, criblage phytochimique, antioxydant.

\section{Phytochemical study and comparative antioxidant activity of the three varieties of Catharanthus roseus (L.) G. Don}

\begin{abstract}
Catharanthus roseus (L.) G. Don, is a medicinal plant widely used in the treatment of diabetes, hypertension, cancer, and effects of oxidative stress. C. roseus exists in three varieties ("rosea", "alba" and "hybrid") depending on the colour of the flowers. This study focused on phytochemical screening and in vitro
\end{abstract}


antioxidant activity (DPPH and FRAP) of the hydro-ethanol extract of the three varieties. The tests were carried out on leaves, flowers, and roots extracts. The presence of alkaloids, phenols, flavonoids, tannins, saponins, sterols, terpenes, and quinines were noted. However, the absence of reducing sugars, flavonoids, and saponins in the roots and quinines in the leaves was observed. Comparative analysis of DPPH radical scavenging showed that extracts from the flowers and roots of the hybrid were more active, respectively at $130.023 \pm 3.2$ and $131.189 \pm 1.8 \mathrm{mg} \mathrm{EqQ} / \mathrm{g}$. FRAP values of the same samples were $175.166 \pm 9.28$ and $121.311 \pm 7.8 \mathrm{mg} \mathrm{EqAA} / \mathrm{g}$ dry matter. For the leaves, the best DPPH value of $65.746 \pm 2.5 \mathrm{mg} \mathrm{EqQ} / \mathrm{g}$ was attributed to "rosea" while the FRAP value of $139.935 \pm 2.57 \mathrm{mg}$ EqAA/g was for "alba". These interesting antioxidant activities of the "hybrid" variety may be useful against oxidative stress pathologies. The current study might then promote the continuing identification and characterization of new bioactive compounds for a development of Catharanthus roseus.

(C) 2020 International Formulae Group. All rights reserved.

Keywords: Catharanthus roseus, varieties, phytochemical screening, antioxidant.

\section{INTRODUCTION}

Bien que la médecine conventionnelle ait connu des avancées spectaculaires, entre 80 et $90 \%$, voire plus, de la population mondiale fait recours à la médecine traditionnelle aujourd'hui (Jiofack et al., 2010). Ce retour vers les plantes est d'autant plus tentant quand on prend en considération les inquiétudes liées aux effets nocifs des médicaments chimiques, la recherche d'une vie saine, la pénurie et le coût élevé des soins de santé. L'efficacité avérée des plantes dans le traitement de nombre de pathologies a amené les chercheurs à se pencher sur les propriétés pharmacologiques de ces plantes. Au Togo, plusieurs plantes sont utilisées dans la pharmacopée; c'est le cas de Catharanthus roseus communément appelé « Pervenche de Madagascar » constituée de trois variétés "rosea", "alba" et "hybride" selon la couleur des fleurs (Nejat et al., 2015). La décoction des feuilles, fleurs ou racines broyées en cocktail est utilisée comme antimitotique, anti-inflammatoire, vermifuge, diurétique, antipaludique, pour soigner l'asthme et le diabète (Radji et Kokou, 2013). Elle est également utilisée comme astringent, diurétique et remède pour la toux (Nejat et al., 2015). La décoction de cette plante est conseillée contre le diabète et l'hypertension artérielle (Adomou et al., 2012; Kouadio et al., 2016) alors que le broyat des feuilles est utilisé pour traiter l'hémorroïde (Fundiko et al., 2017).

En outre, plus de 150 alcaloïdes ont été isolés des extraits de $C$. roseus dont la vinblastine et la vincristine. En effet, la vinblastine et la vincristine sont les premiers agents anticancéreux en usage clinique d'origine végétale utilisés en combinaison avec d'autres médicaments chimio-thérapeutiques pour divers traitements du cancer (Pham et al., 2017; Nejat et al., 2015). Des études plus récentes sur $C$. roseus ont révélés la présence des alcaloïdes, des flavonoïdes, des phénols, des carbohydrates, des saponines, des quinones, des tannins et des glycosides cardiaques dans ses divers extraits (Rani et al., 2017). De plus, une forte activité antiradicalaire due aux nouvelles combinaisons phénoliques dans la graine, la tige, la feuille et le pétale, et aux acides organiques dans la racine de $C$. roseus (Pereira et al., 2010) a été également révélée. La cause connue des pathologies tels que le cancer, les maladies cardiovasculaires, l'athérosclérose, l'hypertension, de l'ischémie (blessure de perfusion), le diabète sucré, les maladies neurodégénératives (maladie d'Alzheimer et de Parkinson), l'arthrite rhumatoïde et du vieillissement étant le stress oxydant (Valko et al., 2007), l'utilisation de produits riches en phénols et flavonoïdes qui se sont révélés pour leurs capacités antioxydantes pourrait jouer un rôle important dans la prévention de ces pathologies (Favier, 2003). Cette étude visait à identifier les grands groupes chimiques et à évaluer l'activité antioxydante des extraits hydro-éthanoliques des trois variétés de $C$. roseus récoltées au Togo. Ainsi la réalisation de ce travail constituera une base pouvant aboutir à la connaissance des principaux constituants de chacune des variétés pour une 
valorisation de cette espèce végétale à travers une meilleure prise en charge de ces pathologies.

\section{MATERIEL ET METHODES Matériel végétal}

Les trois variétés de $C$. roseus ont été récoltées en août 2019 à Amakpapé (6 46'51,44772” N 1 ${ }^{\circ} 10^{\prime} 43,21596$ ” E) dans la préfecture de Haho à environ $75 \mathrm{Km}$ de Lomé pendant la période de floraison et de fructification après trois mois de maturité. Les trois variétés, à savoir "rosea", "alba" et "hybride", ont été authentifiées au Laboratoire de Botanique et d'Ecologie Végétale du département de botanique de la Faculté des Sciences de l'Université de Lomé sous les numéros TOGO 15512, 15513 et 15514, respectivement. Les feuilles, fleurs et racines de chaque variété ont été ensuite séchées à l'abri de la lumière finement broyées puis conservées pour des analyses et traitements ultérieures.

\section{Réactifs chimiques}

Les réactifs utilisés sont : le DPPH $(1,1-$ diphényl-2-picryl-hydrazyle), l'acide ascorbique, la quercétine, le trichlorure d'aluminium $\left(\mathrm{AlCl}_{3}\right)$, le trichlorure de fer III $\left(\mathrm{FeCl}_{3}\right)$ et la solution de ferricyanure de potassium $\mathrm{K}_{3} \mathrm{Fe}(\mathrm{CN})_{6}$ proviennent du fournisseur Carlo Erba. Le carbonate de sodium $\left(\mathrm{Na}_{2} \mathrm{CO}_{3}\right)$, le réactif de FolinCiocalteu, l'acide gallique, quant à eux sont achetés chez Sigma Aldrich. L'éthanol, le méthanol et l'eau distillée ont été utilisés comme solvants.

\section{Méthodes \\ Préparation des extraits}

A $100 \mathrm{~g}$ de poudre des feuilles, ou des fleurs ou encore des racines de chaque variété, a été ajouté $100 \mathrm{~mL}$ d'un mélange éthanol-eau (80:20 v/v); le mélange obtenu a été laissé sous agitation pendant 72 heures à la température ambiante. L'extrait hydro-alcoolique a été récupéré après filtration du mélange à l'aide d'un papier filtre, l'extrait a été concentré par évaporation à l'aide d'un évaporateur rotatif
(BÜCHI). Les extraits ont été récupérés et conservés pour les différents tests réalisés.

\section{Screening phytochimique}

Les grands groupes chimiques tels que les alcaloïdes, les phénols, les flavonoïdes, les tanins, les saponosides, les stérols, les terpènes et les quinines de chaque variété ont été déterminés par une approche basée sur des tests de solubilité, des réactions de coloration et de précipitation selon les méthodes décrites dans la littérature (Kabesh et al., 2015; Rani et al., 2017).

\section{Dosage des phénols totaux}

La méthode utilisée est celle de Shetty et al. (1995) avec quelques modifications. A $0,05 \mathrm{~mL}$ de l'extrait, on ajoute $0,25 \mathrm{~mL}$ de

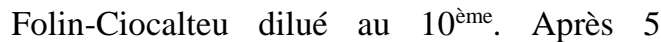
minutes de réaction, $0,2 \mathrm{~mL}$ de carbonate de sodium $\left(\mathrm{Na}_{2} \mathrm{CO}_{3}\right)$ à $145 \mathrm{~g} / \mathrm{L}$ ont été ajoutés au mélange. Le mélange a été bien homogénéisé et l'ensemble a été incubé pendant 30 minutes à température ambiante à l'abri de la lumière et la lecture a été effectuée à $765 \mathrm{~nm}$ contre un blanc à l'aide d'un spectrophotomètre UVVisible 5100B SPECTRO PHOTOMETER. La gamme étalon de 10 à $100 \mu \mathrm{g} / \mathrm{mL}$ a été préparée dans les mêmes conditions que l'extrait. La courbe d'étalonnage a été tracée en utilisant les différentes concentrations de l'acide gallique et les résultats ont été exprimés en milligramme équivalent d'acide gallique par gramme d'extrait sec (mg EqAG/g ES).

\section{Dosage des flavonoüdes}

La méthode utilisée est celle d'Ayoola et al. (2008) avec quelques modifications. À 2 $\mathrm{mL} \mathrm{d} \mathrm{d}^{\prime} \mathrm{AlCl}_{3}$ à $2 \%(\mathrm{~m} / \mathrm{v})$ dans du méthanol pur, est ajouté $2 \mathrm{~mL}$ d'extrait $(1 \mathrm{mg} / \mathrm{mL})$ également dans du méthanol. Le mélange a été incubé pendant 10 minutes à l'abri de la lumière et l'absorbance a été mesurée à $415 \mathrm{~nm}$ contre un blanc à l'aide d'un spectrophotomètre UVVisible. La gamme étalon de 10 à $100 \mu \mathrm{g} / \mathrm{mL}$ a été préparée dans les mêmes conditions que l'extrait. La courbe d'étalonnage a été tracée en utilisant les différentes concentrations de la quercétine et les résultats ont été exprimés en milligramme équivalent de quercétine par gramme d'extrait sec (mg EqQ/g ES). 
Test de réduction du radical DPPH (2,2'diphényl-1-picrylhydrazyle)

L'activité antiradicalaire des différents extraits a été évaluée en utilisant le 2,2'diphényl-1-picrylhydrazyle (DPPH) comme un radical libre relativement stable, selon la méthode décrite par Bakoma et al. (2019) avec quelques modifications. Cent microlitres (100 $\mu \mathrm{L})$ d'extrait ont été ajoutés à $2 \mathrm{~mL}$ DPPH $(0,004 \%$ préparée dans du méthanol). Le mélange a été homogénéisé par un vortex et la lecture de l'absorbance a été faite au spectrophotomètre à $517 \mathrm{~nm}$ après 30 minutes d'incubation à température ambiante et à l'abri de la lumière. Trois essais ont été effectués pour chaque échantillon. Le contrôle positif est représenté par une solution d'un antioxydant standard (Quercétine 100 à $1000 \mathrm{mg} / \mathrm{mL}$ ) dont l'absorbance a été mesuré dans les mêmes conditions que les échantillons et pour chaque concentration, le test est répété trois fois. La courbe d'étalonnage a été tracée en utilisant les différentes concentrations de quercétine et les résultats sont exprimés en milligramme d'équivalent de quercétine par gramme d'extrait sec (mg EqQ/g ES).

\section{Test FRAP (Ferric Reducing Antioxidant Power)}

Le pouvoir réducteur du fer $\left(\mathrm{Fe}^{3+}\right)$ dans les extraits est déterminé selon la méthode de Vijayalakshmi et Ruckmani (2016) avec quelques modifications. Elle est basée sur la réduction de $\mathrm{Fe}^{3+}$ du complexe ferricyanure à la forme du fer ferreux $\left(\mathrm{Fe}^{2+}\right)$. Par conséquent, la teneur de $\mathrm{Fe}^{2+}$ peut être évaluée en mesurant l'augmentation de la densité de la couleur bleue dans le milieu réactionnel à $700 \mathrm{~nm}$. A $0,5 \mathrm{~mL}$ d'extrait, on mélange $1,25 \mathrm{~mL}$ d'une solution tampon phosphate $0,2 \mathrm{M}(\mathrm{pH} 6.6)$ et $1,25 \mathrm{~mL}$ d'une solution de ferricyanure de potassium $\mathrm{K}_{3} \mathrm{Fe}(\mathrm{CN})_{6}$ à $1 \%$. L'ensemble est incubé au bain-marie à $50{ }^{\circ} \mathrm{C}$ pendant $20 \mathrm{~min}$, ensuite $1,25 \mathrm{~mL}$ d'acide trichloracétique à $10 \%$ sont ajoutés pour stopper la réaction et les tubes sont centrifugés à $3000 \mathrm{rpm}$ pendant $10 \mathrm{~min}$. Un virgule vingt-cinq millilitres $(1,25 \mathrm{~mL}) \mathrm{du}$ surnageant est mélangé avec $1,25 \mathrm{~mL}$ d'eau distillée et $0,25 \mathrm{~mL}$ d'une solution aqueuse de $\mathrm{FeCl}_{3}$ à $0,1 \%$. Le mélange a été homogénéisé par un vortex et la lecture de l'absorbance a été faite au spectrophotomètre UV-VIS à $700 \mathrm{~nm}$ après 10 minutes d'incubation à température ambiante. Trois essais ont été effectués pour chaque échantillon. Le contrôle positif est représenté par une solution d'acide ascorbique (antioxydant standard) dont l'absorbance a été mesuré dans les mêmes conditions que les échantillons. La courbe d'étalonnage a été tracée en utilisant les différentes concentrations d'acide ascorbique et les résultats sont exprimés en $\mathrm{mg}$ d'équivalent d'acide ascorbique par gramme d'extrait sec (mg EqAA/g ES).

\section{Analyses statistiques}

Les résultats de dosages (phénols et flavonoïdes totaux) et des tests antioxydants (DPPH et FRAP) ont été traités grâce aux logiciels Excel 2016 et $\mathrm{GraphPad}^{\circledR} 6.05$ puis exprimés en moyenne \pm ESM (Erreur Standard de la Moyenne).

\section{RESULTATS}

\section{Screening phytochimique}

Le screening phytochimique (Tableau 1) des extraits hydro-éthanoliques a révélé la présence des alcaloïdes, des phénols, des flavonoïdes, des tanins, des saponosides, des stérols, des terpènes et des quinines dans la plupart des échantillons. Cependant, on a noté l'absence de flavonoïdes et de saponosides au sein des échantillons de racines ainsi que des quinines dans les feuilles des trois variétés. On a noté également l'absence des sucres réducteurs dans les organes des trois variétés.

\section{Dosage des phénols totaux}

Les teneurs en phénols totaux des extraits hydro-éthanoliques des organes (fleurs, feuilles et racines) des trois variétés de C. roseus ont été déterminées à partir de la droite de régression linéaire $(\mathrm{Y}=0,01402 \mathrm{X}+$ 0,$\left.02472 ; \quad R^{2}=0,9969\right)$ de la courbe d'étalonnage de l'acide gallique. Ainsi, les teneurs en phénols totaux obtenues sont respectivement de $41,865 \pm 0,512$; $29,312 \pm 0,454$ et $22,750 \pm 0,292 \mathrm{mg}$ EqAG/g ES dans les fleurs, feuilles et racines de "rosea"; $11,932 \pm 0,109 ; 18,874 \pm 0,171$ et $14,737 \pm 0,578$ $\mathrm{mg}$ EqAG/g ES dans les fleurs, feuilles et racines de "alba" et enfin de 43,315 $\pm 1,565$; $19,754 \pm 0,930$ et $26,173 \pm 0,671 \mathrm{mg} \mathrm{EqAG} / \mathrm{g}$ 
ES dans les fleurs, feuilles et racines de la variété "hybride". La teneur comparée en phénols des organes est illustrée sur la Figure 1.

\section{Dosage des flavonoïdes totaux}

Les teneurs en flavonoïdes totaux des extraits hydro-éthanoliques des organes (fleurs, feuilles et racines) des trois variétés de C. roseus ont été déterminées à partir de la droite de régression linéaire $(\mathrm{Y}=0,01589 \mathrm{X}$ 0,$1087 ; R^{2}=0,9838$ ) de la courbe d'étalonnage de la quercétine. Les teneurs obtenues sont respectivement de 75,550 $\pm 2,13 ; 70,424 \pm 3,55$ et $24,399 \pm 0,17 \mathrm{mg} \mathrm{EqQ/g}$ ES dans les fleurs, feuilles et racines de "rosea"; 80,891 $\pm 4,53$; $101,345 \pm 2,19$ et $18,316 \pm 0,54 \mathrm{mg} \mathrm{EqQ} / \mathrm{g}$ ES dans les fleurs, feuilles et racines de "alba" et enfin de $58,173 \pm 0,81 ; \quad 149,971 \pm 2,54$ et $15,274 \pm 0,44 \mathrm{mg} \mathrm{EqQ} / \mathrm{g}$ ES dans les fleurs, feuilles et racines de la variété "hybride". En plus, la Figure 2 présente ces résultats comparés des teneurs en flavonoïdes totaux.

Test de réduction du radical DPPH (2,2'diphényl-1-picrylhydrazyle)

Le pouvoir réducteur du radical 2,2'diphényl-1-picrylhydrazyle (DPPH) a été déterminé à partir de la droite de régression linéaire d'équation $\mathrm{Y}=-0,004290 \mathrm{X}+0,8188$; $\mathrm{R}^{2}=0,9946$. L'activité antioxydante des extraits était respectivement de 78,8 $\pm 1,9$; $87,308 \pm 2,02$ et $101,352 \pm 2,8 \mathrm{mg} \mathrm{EqQ} / \mathrm{g}$ ES pour les fleurs, feuilles et racines de "rosea"; $57,413 \pm 1,5 ; 65,746 \pm 2,5$ et $101,177 \pm 3,2 \mathrm{mg}$ $\mathrm{EqQ} / \mathrm{g}$ ES pour les fleurs, feuilles et racines de "alba" et de $130.023 \pm 3,2 ; 63,531 \pm 1,9$ et $131,189 \pm 1.8 \mathrm{mg} \mathrm{EqQ} / \mathrm{g}$ ES pour les fleurs, feuilles et racines de la variété "hybride". En outre, la Figure 3 présente une illustration comparée des résultats de DPPH.

\section{Test FRAP (Ferric Reducing Antioxidant Power)}

Le pouvoir réducteur du fer (FRAP) a été déterminé à partir de la droite de régression linéaire d'équation $\mathrm{Y}=0,002810 \mathrm{X}+0,09945$; $\mathrm{R}^{2}=0,9912$. Les pouvoirs réducteurs des extraits étaient respectivement de 103,636 $\pm 9,0$; $77,894 \pm 11,65$ et $126,649 \pm 5,1 \mathrm{mg}$ EqAA/g ES pour les fleurs, feuilles et racines de "rosea"; $142,426 \pm 12,04 ; \quad 139,935 \pm 2,57 \quad$ et $144,205 \pm 10,42 \mathrm{mg}$ EqAA/g ES pour les fleurs, feuilles et racines de "alba" et de $175,166 \pm 9,28,107,788 \pm 8,43$ et de $121,311 \pm 78$ mg EqAA/g ES dans le même ordre pour la variété "hybride". La Figure 4 illustre une présentation comparée des résultats du test FRAP.

Tableau 1: Screening phytochimique.

\begin{tabular}{cccccccccc}
\hline & \multicolumn{8}{c}{ Variétés de catharanthus roseus } \\
\cline { 2 - 11 } & \multicolumn{7}{c}{ Rosea } & \multicolumn{7}{c}{ Alba } & \multicolumn{3}{c}{ Hybride } \\
\cline { 2 - 11 } & Fl & Fe & Rac & Fl & Fe & Rac & Fl & Fe & Rac \\
\hline $\begin{array}{c}\text { Alcaloïdes } \\
\text { (Mayer) }\end{array}$ & + & + & + & + & + & + & + & + & + \\
\hline Flavonoïdes & + & + & - & + & + & - & + & + & - \\
\hline Phénols & + & + & + & + & + & + & + & + & + \\
\hline Tanins & + & + & + & + & + & + & + & + & + \\
\hline Saponines & + & + & - & + & + & - & + & + & - \\
\hline $\begin{array}{c}\text { Stérols et } \\
\text { terpènes }\end{array}$ & + & + & + & + & + & + & + & + & + \\
\hline $\begin{array}{c}\text { Sucres } \\
\text { réducteurs }\end{array}$ & - & - & - & - & - & - & - & - & - \\
\hline Quinines & + & - & + & + & - & + & + & - & + \\
\hline
\end{tabular}

+: présence ; -: absence; Fl: fleurs; Fe: feuilles; Rac: racines 


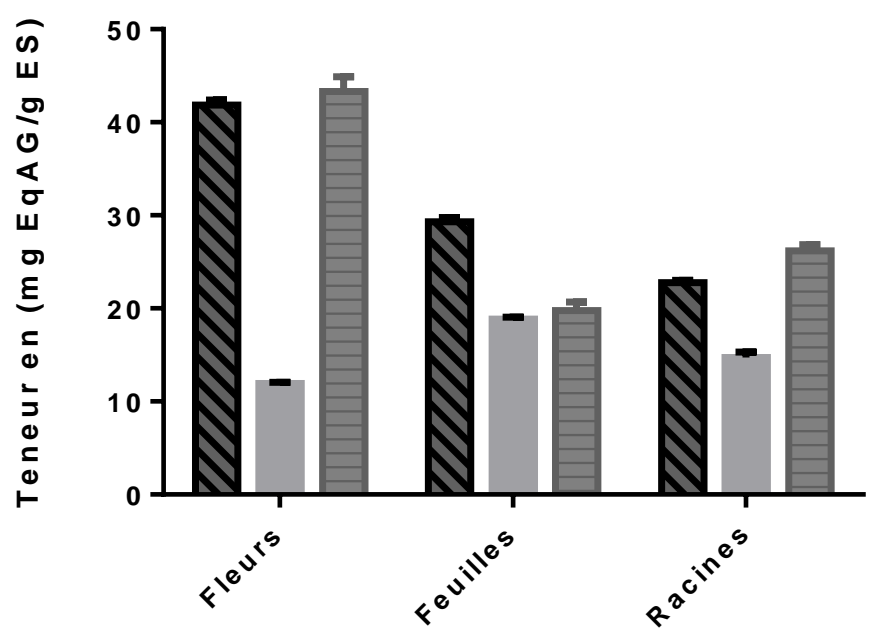

Figure 1 : Teneurs en phénols totaux des extraits des organes des variétés de C.roseus.

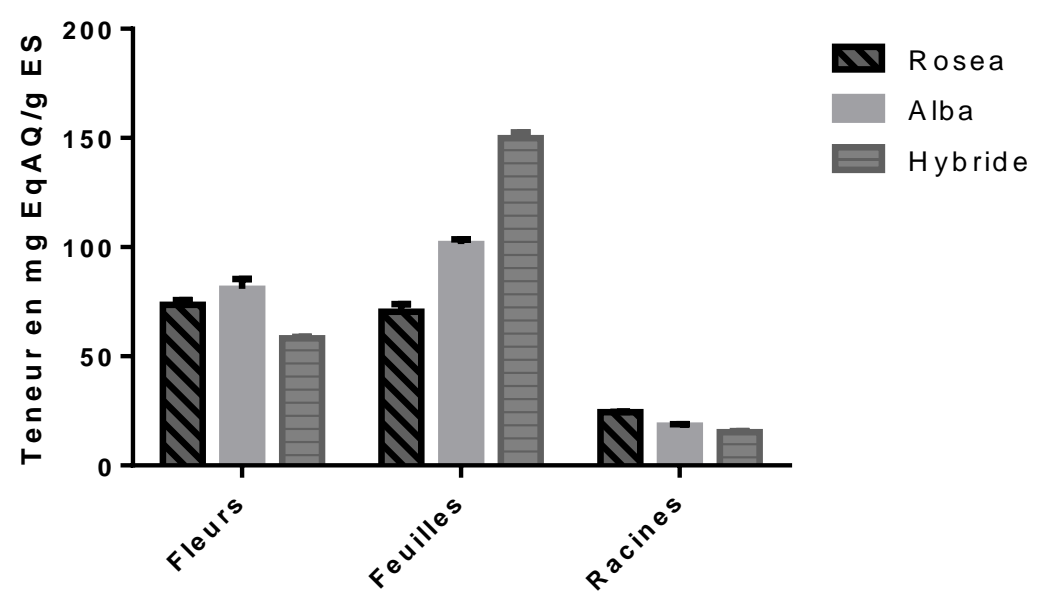

Figure 2 : Teneurs en flavonoïdes totaux des extraits des organes des variétés de C.roseus.

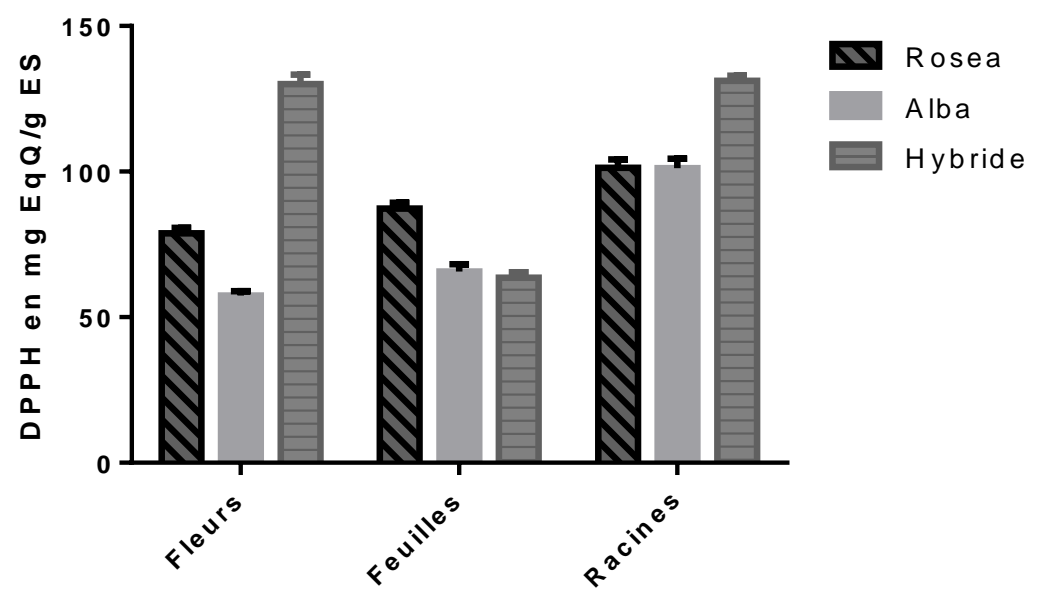

Figure 3 : Pouvoir anti-radicalaire des extraits des organes des variétés de C. roseus. 


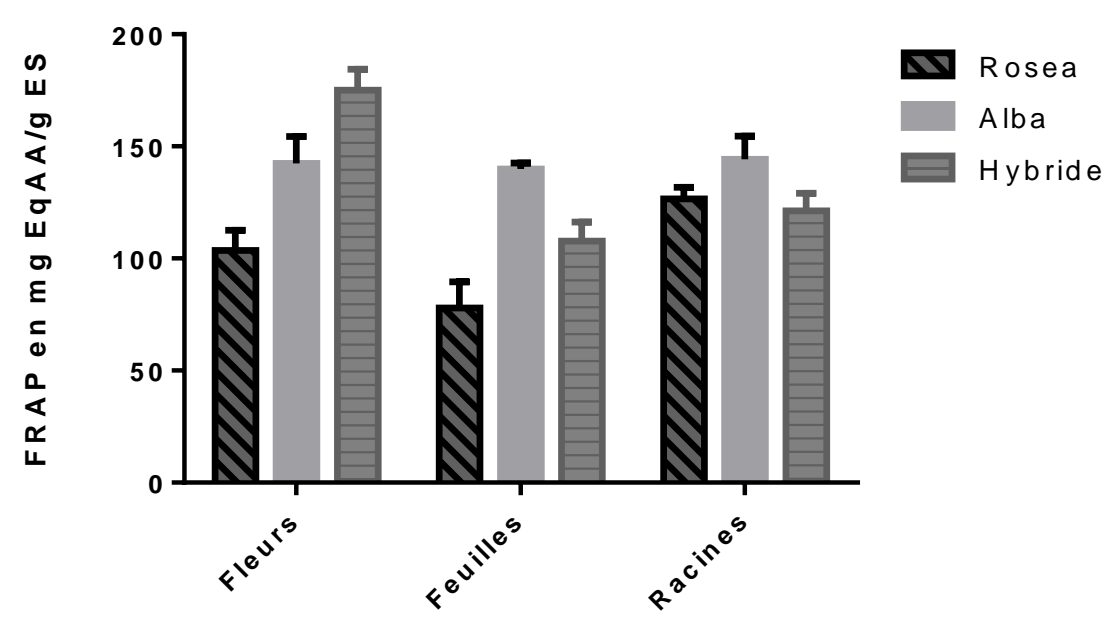

Figure 4 : Pouvoir réducteur des extraits des organes des variétés de $C$. roseus.

\section{DISCUSSION}

L'utilisation d'un mélange hydroéthanolique (solvant) nous a permis d'obtenir les extraits d'organes (fleurs, feuilles et racines) des trois variétés de $C$. roseus, extraits qui renferment des composés phénoliques, les tanins, les flavonoïdes et les saponosides. Ces composés sont tous connus comme étant les principaux constituants des plantes réputées pour leur activités antioxydantes (Favier, 2003).

Les tests phytochimiques réalisés sur les extraits des différents organes des trois variétés de $C$. roseus ont révélé la présence d'alcaloïdes, de phénols, de flavonoïdes, de tanins, de saponines, de stérols, de terpènes et de quinines. Cependant, on note l'absence de flavonoïdes et de saponines dans les racines ainsi que de quinines dans les feuilles des trois variétés. On a également noté l'absence des sucres réducteurs dans les organes des trois variétés à savoir "rosea", "alba" et "hybride". Les travaux de Kabesh et al. (2015), portant sur l'espèce "rosea", ont révélés pratiquement les mêmes métabolites secondaires dans les feuilles. Ces résultats corroborent également ceux de Rani et al. (2017), Kabesh et al. (2015), et de Govindasamy et Srinivasan (2012); travaux dans lesquels il a été noté l'absence de stérols et de saponosides dans l'extrait des feuilles de $C$. roseus. Cette différence pourrait s'expliquer par les facteurs climatiques et géographiques. La richesse en ces grands groupes de composés actifs devrait justifier l'usage traditionnel de cette plante dans les soins de nombreuses maladies telles que le diabète (Gbekley et al., 2015), l'hypertension (Karou et al., 2011) ; comme antimitotique, anti-inflammatoire, vermifuge, diurétique, antipaludique, antiasthmatique, antipaludique (Radji et Kokou, 2013).

Les résultats du dosage des phénols totaux ont montré que pour les fleurs, les extraits de la variété "hybride" ont donné une teneur en phénols élevée suivit de "rosea" puis "alba" (Figure 1). Pour les extraits des feuilles, la variété "rosea" a présenté une teneur élevée suivie de la variété "hybride" puis ensuite de "alba". Au niveau des racines, la teneur en phénols a suivi une tendance similaire à celle des fleurs, c'est-à-dire "hybride", "rosea" puis "alba" (Figure 1). Cette disparité des teneurs en phénols au sein des différents organes de $C$. roseus pourrait s'expliquer par les facteurs génétiques (Dragovicuzelac et al., 2007) et par l'influence des pigments qui ont généralement des effets sur la croissance des variétés "rosea" et "alba" de C. roseus (Jaleel et al., 2008).

En outre, les teneurs en flavonoïdes dans les extraits d'organes de $C$. roseus sont 
également présentés sur la Figure 2. Ces résultats montrent que les extraits des fleurs de la variété "alba" présentent une teneur en flavonoïdes élevée suivie de la variété "rosea" puis "hybride". Pour les extraits des feuilles, la variété "hybride" présente une meilleure teneur suivie de la variété "alba" puis enfin de "rosea". Les extraits des racines présentent une teneur élevée à la variété "rosea" suivie de la variété "alba" puis enfin de "hybride". Il faut également relever que la teneur en flavonoïdes est plus faible dans les racines comparativement aux autres organes (fleurs et feuilles). Ces faibles teneurs en flavonoïdes au niveau des racines comparativement aux autres organes pourraient s'accorder avec leur absence dans les échantillons de racines lors du screening phytochimique (Tableau 1). Cette différence de teneurs en flavonoïdes dans les organes de $C$. roseus devrait être due, comme pour les phénols totaux, aux facteurs génétiques (Bouzid et al., 2011). Aussi, les extraits hydro-éthanoliques des feuilles de $C$. roseus ont globalement donné une teneur élevée en flavonoïdes par rapport aux fleurs et racines. Cette teneur élevée en flavonoïdes dans les feuilles pourrait se justifier par le fait que les feuilles sont plus exposées au soleil que les autres organes de la plante. En effet, les flavonoïdes assureraient la protection des tissus de la plante contre les effets nocifs des rayons solaires (Tattini et al., 2006).

Le test anti-radicalaire au DPPH des extraits des organes de C. roseus (Figure 3) ont montré une intéressante capacité antiradicalaire des échantillons de fleurs et de racines de l'hybride. L'extrait des feuilles de la variété "rosea" a, quant à lui, offert une meilleure capacité anti-radicalaire par rapport aux feuilles de "alba" et de "hybride". Aussi, les extraits hydro-éthanoliques des racines des trois variétés de $C$. roseus ont présenté les plus fortes propriétés de piégeage du DPPH. Cette intéressante capacité anti-radicalaire des extraits des racines pourrait s'expliquer par la présence des acides organiques, réputés pour leurs activité antioxydante dans les racines de C. roseus (Pereira et al., 2010).
En outre, l'évaluation du pouvoir réducteur du fer (FRAP) des extraits hydroéthanolique des variétés de C. roseus (Figure 4) ont montré que l'extrait des fleurs de "hybride" a une capacité de réduction du fer intéressante par rapport à "rosea" et "alba" dans cet ordre. L'extrait des feuilles de la variété "alba" a donné une meilleure capacité de réduction du fer par rapport à l'espèce "hybride" suivi de "rosea". Les racines de la variété "alba" ont quant à elles donné une importante capacité de réduction du fer. En effet, d'après le Tableau 2, le pouvoir réducteur de l'ion ferrique des fleurs de la variété "hybride" est meilleur par rapport aux autres organes. Ceci pourrait s'expliquer par une teneur élevée de flavonoïdes dans cet extrait. De plus, les capacités antioxydantes des extraits obtenus par la méthode au DPPH sont nettement inférieures à celles de la méthode FRAP. Le rôle très important des phénols et flavonoïdes dans la chélation des métaux de transitions impliqués dans la réaction de Fenton (Valko et al., 2007) justifierait cette tendance.

\section{Conclusion}

Cette étude réalisée sur les extraits hydro-éthanoliques des organes de C. roseus montre que les extraits des variétés "alba" et "hybride" sont plus actifs que celle de " rosea " bien que celle de la variété "hybride" est légèrement meilleure. En effet, cette activité des différents extraits serait en partie liée aux phénols et flavonoïdes totaux contenus dans ces extraits. Une étude ultérieure dans le but $\mathrm{d}$ 'isoler et identifier des molécules bioactives à activités antioxydantes devrait permettre de mieux cerner la nature des principes actifs pour une utilisation rationnelle de cette ressource naturelle en vue d'une valorisation par des médicaments traditionnels améliorés.

\section{CONFLIT D'INTERETS}

Les auteurs déclarent qu'ils n'ont aucun conflit d'intérêts.

\section{CONTRIBUTIONS DES AUTEURS}

HK, OS, GWT et SS ont participé à toutes les phases de cette étude. 


\section{REMERCIEMENTS}

Les auteurs remercient le Laboratoire de botanique et d'écologie végétale du département de botanique, Faculté des Sciences, l'Université de Lomé, pour avoir permis l'identification des espèces étudiées.

\section{REFERENCES}

Adomou A, Yedomonhan H, Djossa B, Legba S, Oumorou M, Akoegninou A. 2012. Etude Ethnobotanique des plantes médicinales vendues dans le marché d'Abomey-Calavi au Bénin. Int. J. Biol. Chem. Sci., 6(2): 745-772. DOI: https://doi.org/10.4314/ijbcs.v6i2.18.

Ayoola G, Coker H, Adesegun S, AdepojuBello A, Obaweya K, Ezennia E, Atangbayila T. 2008. Phytochemical Screening and Antioxidant Activities of Some Selected Medicinal Plants Used for Malaria Therapy in Southwestern Nigeria. Trop J Pharm Res., 7(3) : 10191024.

DOI:

https://doi.org/10.4314/tjpr.v7i3.14686.

Bakoma B, Sanvee S, Metowogo K, Potchoo Y, Eklu-gadegbeku K, Aklikokou K, Gbeassor M. 2019. Phytochemical Study and Biological Activities of HydroAlcoholic Extract of the Leaves of Bridelia ferruginea Benth and its Fractions. Pharmacogn. J., 11(1): 141145.

DOI: https://doi.org/10.5530/pj.2019.1.23.

Bouzid W, Yahia M, Abdeddaim M, Aberkane MC. 2011. Evaluation de l'activité antioxydante et antimicrobienne des extraits de l'aubepine monogyne. Leban. Sci. J., 12(1): 59-69.

Dragovicuzelac V, Levaj B, Mrkic V, Bursac D, Boras M. 2007. The content of polyphenols and carotenoids in three apricot cultivars depending on stage of maturity and geographical region. Food Chem., 102(3): 966-975. DOI: https://doi.org/10.1016/j.foodchem.2006. 04.001 .

Favier A. 2003. Le stress oxydant : Intérêt conceptuel et expérimental dans la compréhension des mécanismes des maladies et potentiel thérapeutique.
Journal de La Société Chimique de France, 108-115.

Fundiko MCC, Mandango MA, Mutambala BM. 2017. Etude ethnobotanique des plantes utilisees dans le traitement des maladies de l'appareil digestif à Kinshassa et ses environs, République Démocratique du Congo. International Journal of Innovation and scientific Research, 31(1): 194-203.

Gbekley EH, Karou DS, Gnoula C, Agbodeka K, Anani K, Tchacondo T, Agbonon A, Batawila K, Simpore J. 2015. Étude ethnobotanique des plantes utilisées dans le traitement du diabète dans la médecine traditionnelle de la région Maritime du Togo. Pan Afr Med J., 20(437). DOI: https://doi.org/10.11604/pamj.2015.20.4 37.5660 .

Govindasamy C, Srinivasan R. 2012. In vitro antibacterial activity and phytochemical analysis of Catharanthus roseus (Linn.) G. Don. Asian Pac. J. Trop. Biomed., 2(1): $\quad 155-158 . \quad$ DOI: https://doi.org/10.1016/S22211691(12)60148-8.

Jaleel CA, Gopi R, Manivannan P, Gomathinayagam $M$, Sridharan $R$, Panneerselvam R. 2008. Antioxidant potential and indole alkaloid profile variations with water deficits along different parts of two varieties of Catharanthus roseus. Colloid Surface B., 62(2): $\quad 312-318 . \quad$ DOI: https://doi.org/10.1016/j.colsurfb.2007.1 0.013 .

Jiofack T, Fokunang C, Guedje N, Kemeuze V, Fongnzossie E, Nkongmeneck BA, Mapongmetsem PM, Tsabang N. 2010. Ethnobotanical uses of medicinal plants of two ethnoecological regions of Cameroon. Int. J. Med. Med. Sci., 2(3): 20.

Kabesh K, Senthilkumar P, Ragunathan R, Kumar R. 2015. Phytochemical Analysis of Catharanthus roseus Plant Extract and its Antimicrobial Activity. Int. J. Pure App. Biosci., 3(2): 162-172.

Karou SD, Tchacondo T, Djikpo Tchibozo MA, Abdoul-Rahaman S, Anani K, 
Koudouvo K, Batawila K, Agbonon A, Simpore J, De Souza C. 2011. Ethnobotanical study of medicinal plants used in the management of diabetes mellitus and hypertension in the Central Region of Togo. Pharm. Biol., 49(12): 1286-97. DOI: https://doi.org/10.3109/13880209.2011.6 21959.

Kouadio B, Djeneb C, Yvette FNB, Basile YA, Cynthia Y, Alain AS, Noël ZG. 2016. Étude ethnobotanique des plantes médicinales utilisées dans le Département de Transua, District du Zanzan (Côte d'Ivoire). J. Anim. Plant Sci., 27(2): 4230-4250.

Nejat N, Valdiani A, Cahill D, Tan YH, Maziah M, Abiri R. 2015. Ornamental Exterior versus Therapeutic Interior of Madagascar Periwinkle (Catharanthus roseus): The Two Faces of a Versatile Herb. Sci. World J., 1-19. DOI: https://doi.org/10.1155/2015/982412.

Pereira DM, Faria J, Gaspar L, Ferreres F, Valentão P, Sottomayor M, Andrade PB. 2010. Exploiting Catharanthus roseus roots: Source of antioxidants. Food Chem., 121(1): 56-61. DOI: https://doi.org/10.1016/j.foodchem.2009. 12.002 .

Pham HNT, Vuong QV, Bowyer MC, Scarlett CJ. 2017. Effect of extraction solvents and thermal drying methods on bioactive compounds and antioxidant properties of Catharanthus roseus (L.) G. Don (Patricia White cultivar). J Food Process Preserv., 41(5): e13199. DOI: http://doi.wiley.com/10.1111/jfpp.13199.

Radji R, Kokou K. 2013. Classification et valeurs thérapeutiques des plantes ornementales du Togo. VertigO, 13(3). DOI: https://doi.org/10.4000/vertigo.14519.

Rani J, Kapoor M, Kaur R. 2017. In-vitro antibacterial activity and phytochemical screening of crude extracts of Catharanthus roseus L. (G.) Don. Agric. Sci. Digest., 37(2) 2017: 106-111. DOI: https://doi.org/10.18805/asd.v37i2.7983.

Shetty K, Curtis OF, Levin RE, Witkowsky R, Ang W. 1995. Prevention of Vitrification Aßociated with in vitro Shoot Culture of Oregano. (Origanum vulgare) by Pseudomonas spp. J. Plant Physiol., 147(3-4): 447-451. DOI: https://doi.org/10.1016/S01761617(11)82181-4.

Tattini M, Remorini D, Pinelli P, Agati G, Saracini E, Traversi ML, Massai R. 2006. Morpho-anatomical, physiological and biochemical adjustments in response to root zone salinity stress and high solar radiation in two Mediterranean evergreen shrubs, Myrtus communis and Pistacia lentiscus. New Phytol., 170(4): 779-794. DOI: https://doi.org/10.1111/j.14698137.2006.01723.x.

Valko M, Leibfritz D, Moncol J, Cronin MTD, Mazur M, Telser J. 2007. Free radicals and antioxidants in normal physiological functions and human disease. Int. J. Biochem. Cell Biol., 39(1): 44-84. DOI: https://doi.org/10.1016/j.biocel.2006.07. 001.

Vijayalakshmi M, Ruckmani K. 2016. Ferric reducing anti-oxidant power assay in plant extract. Bangladesh J. Pharmacol., 11(3): $\quad 570 . \quad$ DOI: https://doi.org/10.3329/bjp.v11i3.27663. 